\section{Patient Room Decontamination against Carbapenem-Resistant Enterobacteriaceae and Methicillin-Resistant Staphylococcus aureus Using a Fixed Cycle-Time Ultraviolet-C Device and Two Different Radiation Designs}

The contaminated surface environment of hospital rooms has been demonstrated to be a source of patient-to-patient transmission of multidrug-resistant organisms (MDROs), including methicillin-resistant Staphylococcus aureus (MRSA). ${ }^{1,2}$ MDROs can survive on surfaces for days to weeks (for months in some cases). ${ }^{3}$ Following terminal room cleaning and disinfection, frequent contamination by these pathogens has been reported. ${ }^{1,2}$ Patients admitted to a room where the previous occupant was also colonized or infected with an MDRO are at substantial risk for acquisition of this MDRO. ${ }^{4}$ For these reasons, "no-touch" methods of room disinfection have been evaluated, including devices that emit ultraviolet (UV) light or produce hydrogen peroxide vapor. ${ }^{5}$ Use of UV devices has been demonstrated to reduce healthcare-associated pathogens. ${ }^{5}$

Carbapenem-resistant Enterobacteriaceae (CRE) are a growing problem in the United States and worldwide. ${ }^{6} \mathrm{CRE}$ infections are difficult to treat and have been associated with substantial mortality. Frequent contamination of room surfaces near CRE carriers has been described. ${ }^{7}$ For this reason, we assessed the efficacy of a UV-C device to inactivate MRSA and CRE. We also assessed whether 2 cycles of the device (in which 1 cycle was completed on the right side of the bed and 1 cycle on the left side of the bed) were superior to a single cycle (in which the device was placed at the foot of the bed).

In this study, we evaluated the Optimum UV (Clorox, Pleasanton, CA), a fixed cycle-time UV-C $(254 \mathrm{~nm})$ device with four $157-\mathrm{cm}$ lamps in an unoccupied patient room $\left(24.76 \mathrm{~m}^{2}\right)$. To assess the efficacy of 2 different decontamination methods, the UV-C device was placed either at the end of the bed in the center of room in a single cycle (design A) or at both sides of the bed in the center of room with a single cycle at the right side and another cycle at left side (design B). The cycle time of the device for room decontamination was fixed at 5 minutes. Each design was tested in triplicate. Locations where test Formica sheets were placed were categorized as the UV-C direct group (laser point visible on site) or the UV-C indirect group (laser point not visible on site). Testing was performed using clinical isolates of MRSA and carbapenemresistant Klebsiella pneumoniae (CRKP). A $10-15 \mu \mathrm{L}$ inoculum containing $\sim 10^{6}-10^{7}$ test organisms per replicate organism detection and counting (Rodac) template was spread separately on each Formica sheet using a sterile glass hockey loop. Inoculated Formica sheets were placed and attached with tape in the following 10 locations throughout the room for both designs: (1) the back of the computer (indirect/indirect); (2) the side of the sink counter (indirect/direct); (3) the vertical end of the foot of the bed (direct/indirect); (4) the horizontal top of the bed (direct/direct); (5) the vertical back of the visitor's chair (indirect/indirect); (6) the horizontal counter in front of the refrigerator (direct/direct); (7) the vertical end of the head of the bed (indirect/indirect); (8) the vertical side of the bedside table facing the window (indirect/ indirect); (9) the horizontal top of the toilet rim in the bathroom (indirect/indirect); and (10) the horizontal top of the couch table (direct/direct). After UV-C cycle completion, each Formica sheet was cultured using Rodac plates containing Day-Engley (DE) neutralizing agar. These plates were incubated aerobically at $37^{\circ} \mathrm{C}$ for 48 hours for CRKP and MRSA. The colony-forming units (CFU) of the test organisms on each plate were then counted. When Rodac plates had confluent growth, the number of CFUs per plate was set at 300 for analysis. Statistical significance was determined by paired $t$ test; $P<.05$ was considered significant.

Overall, in an unoccupied patient room, we observed a 5.01- $\log _{10}$ reduction using design $\mathrm{A}$ and a $5.87-\log _{10}$ reduction using design $\mathrm{B}$ for decontamination of CRKP $(P=.0003)$. We observed a $4.61-\log _{10}$ reduction and a $5.06-\log _{10}$ reduction for MRSA $(P=.0418)$ (Table 1). At UV-C direct sites, we measured an additional $0.87-\log _{10}$ reduction using design $\mathrm{B}$ for CRKP $(P=.0028)$ and an additional $0.55-\log _{10}$ reduction for MRSA $(P>.05)$ compared with design $A$. When we assessed the correlation of $\log _{10}$ reduction (in direct line of sight $)$ to distance, there was a strong correlation $(r=-0.826$, $P=.0009$ for CRKP and $\mathrm{r}=-0.842, P=.0006$ for MRSA); however, we detected $\mathrm{a} \geq 4.89-\log _{10}$ reduction for CRKP and a $\geq 4.19-\log _{10}$ reduction for MRSA at the farthest distance $(2.69 \mathrm{~m})$. At UV-C indirect sites, we measured an additional $0.86-\log _{10}$ reduction using design B for CRKP $(P=.0002)$ and an additional $0.38-\log _{10}$ reduction for MRSA $(P=.0113)$ compared to design A. The frequency of too-numerousto-count (TNTC) cultures was as follows: MRSA $42 \%$ (25 of 60 ) and CRKP $32 \%$ (19 of 60). Data for microbial inactivation without TNTC results are shown in the Supplemental Material.

Our study demonstrated that the fixed-time UV-C device evaluated in this study was effective in 5-10 minutes in eliminating $>5-\log _{10}$ MRSA and CRKP when the surfaces were in direct line of sight of the UV-C device and $>4-\log _{10}$ MRSA and CRKP when the surfaces were in indirect line of sight. These results are similar to those we have previously published. ${ }^{8}$ While the use of 2 cycles of the device with placement at either side of the bed for 1 cycle each often 
TAвLE 1. Microbial Reduction in Formica Surfaces Experimentally Contaminated with Multidrug-Resistant Pathogens Using a Fixed Cycle-Time Ultraviolet-C (UV-C) Device and 2 Different Radiation Designs

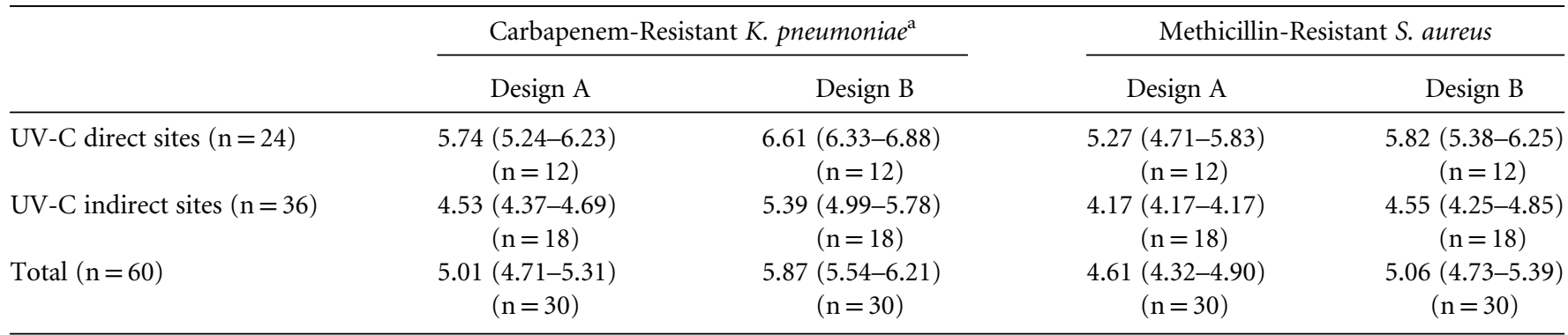

${ }^{\mathrm{a}}$ Values are shown in mean $\log _{10}$ reductions (95\% confidence interval) (No. of samples). Quantitated inoculum was 6.93-log 10 per Rodac template for carbapenem-resistant K. pneumoniae and $6.65-\log _{10}$ per Rodac template for methicillin-resistant $S$. aureus.

Microbial reduction was calculated by subtracting the number $\left(\log _{10}\right)$ of test organisms measured on UV-C cycle completion from quantitated inoculum $\left(\log _{10}\right)$. For design A, the UV-C device was placed at the end of the bed in the center of room with a single cycle (total exposure time: $5 \mathrm{~min}$ ). For design B, the UV-C device was placed at both sides of the bed in the center of room with a single cycle at the right side and another cycle at left side (total exposure time: $10 \mathrm{~min}$ ).

resulted in significantly improved inactivation of the vegetative MDROs studied (ie, MRSA, CRKP), a single cycle was highly effective, and the difference is likely not clinically meaningful. Given the frequency of TNTC cultures, our level of microbial kill should be considered a maximum level.

We are aware of several limitations of this study. First, we only inoculated Formica sheets. Our previous study revealed that CRE survived poorly not only on fabrics but also on hard surfaces such as Formica and steel. ${ }^{9}$ Second, the size of our study limited our ability to perform subgroup analyses such as inactivation in direct versus indirect line of sight.

In this study, we tested a clinical CRE strain because of the global concern regarding its morbidity and mortality. Importantly, the present study demonstrated that UV-C room decontamination was effective against CRE. The role of hospital environmental surfaces in CRE transmission remains controversial: CRE was infrequently $(8 \%)$ isolated from environmental surfaces in rooms housing CRE colonized/ infected patients in our previous study, ${ }^{9}$ while CRE contamination of hospital environment was frequently $(88 \%)$ identified at surrounding sites among patients with this organism by an Israeli study. ${ }^{7}$

In conclusion, the present data and our previous findings have demonstrated that UV-C devices can effectively decontaminate hospital surfaces contaminated with MDROs, including CRE. In this experiment, 2 cycles of a UV-C device were often statistically superior to a single cycle, but both designs are likely to be clinically effective ( $>2-\log _{10}$ reduction) because epidemiologically important pathogens measured in hospital room surfaces were $<100$ CFU/Rodac $\left(<2-\log _{10}\right){ }^{10}$

\section{ACKNOWLEDGMENTS}

Financial support: This study was supported by internal funding from UNC Health Care. H.K. received financial support from the JSPS Postdoctoral Fellowship for Research Abroad.
Potential conflicts of interest: W.A.R. and D.J.W. are consultants for the Clorox Corporation.

\section{Hajime Kanamori, MD, PhD, MPH; ${ }^{1,2}$ William A. Rutala, PhD, MPH; ${ }^{1,2}$ Maria F. Gergen, MT (ASCP); ${ }^{1}$ David J. Weber, MD, MPH ${ }^{1,2}$}

Affiliations: 1. Department of Hospital Epidemiology, University of North Carolina Health Care, Chapel Hill, North Carolina; 2. Division of Infectious Diseases, University of North Carolina School of Medicine, Chapel Hill, North Carolina

Address correspondence to Hajime Kanamori, MD, PhD, MPH, Hospital Epidemiology, UNC Health Care, 1001 West Wing CB \#7600, 101 Manning Drive, Chapel Hill, NC 27514 (kanamori@med.unc.edu).

Received December 29, 2015; accepted March 15, 2016; electronically published April 13, 2016

Infect Control Hosp Epidemiol 2016;37:994-996

(C) 2016 by The Society for Healthcare Epidemiology of America. All rights reserved. 0899-823X/2016/3708-0020. DOI: 10.1017/ice.2016.80

\section{SUPPLEMENTARY MATERIAL}

To view supplementary material for this article, please visit http://dx.doi.org/10.1017/ice.2016.80

\section{REFERENCES}

1. Weber DJ, Rutala WA, Miller MB, Huslage K, Sickbert-Bennett E. Role of hospital surfaces in the transmission of emerging health care-associated pathogens: norovirus, Clostridium difficile, and Acinetobacter species. Am J Infect Control 2010;38: S25-S33.

2. Weber DJ, Anderson D, Rutala WA. The role of the surface environment in healthcare-associated infections. Curr Opin Infect Dis 2013;26:338-344.

3. Kramer A, Schwebke I, Kampf G. How long do nosocomial pathogens persist on inanimate surfaces? A systematic review. BMC Infect Dis 2006;6:130.

4. Otter JA, Yezli S, Salkeld JA, French GL. Evidence that contaminated surfaces contribute to the transmission of hospital 
pathogens and an overview of strategies to address contaminated surfaces in hospital settings. Am J Infect Control, 41:S6-S11.

5. Weber DJ, Rutala WA. Understanding and preventing transmission of healthcare-associated pathogens due to the contaminated surface environment. Infect Control Hosp Epidemiol 2013;34: 449-452.

6. 2012 CRE toolkit-guidance for control of carbapenem-resistant Enterobacteriaceae (CRE). Centers for Disease Control and Prevention website. http://www.cdc.gov/hai/organisms/cre/ cre-toolkit/index.html. Accessed November 30, 2015.

7. Lerner A, Adler A, Abu-Hanna I, et al. Environmental contamination by carbapenem-resistant Enterobacteriaceae. J Clin Microbiol 2013;51:177-181.

8. Rutala WA, Gergen MF, Tande BM, Weber DJ. Room decontamination using an ultraviolet-C device with short ultraviolet exposure time. Infect Control Hosp Epidemiol 2014; 35:1070-1072.

9. Weber DJ, Rutala WA, Kanamori H, Gergen MF, Sickbert-Bennett EE. Carbapenem-resistant Enterobacteriaceae: frequency of hospital room contamination and survival on various inoculated surfaces. Infect Control Hosp Epidemiol 2015;36:590-593.

10. Huslage K, Rutala WA, Gergen MF, Sickbert-Bennett EE, Weber DJ. Microbial assessment of high-, medium-, and low-touch hospital room surfaces. Infect Control Hosp Epidemiol 2013;34:211-212. 